Pacific Journal of Mathematics

UNIFORM REPRESENTATIONS OF CONGRUENCE SCHEMES 


\title{
UNIFORM REPRESENTATIONS OF CONGRUENCE SCHEMES
}

\author{
Joel Berman AND George Grätzer
}

A congruence scheme $\Sigma$ is a finite sequence of polynomials. A nontrivial equational class $K$ is a representation of $\Sigma$ iff the principal congruences in $K$ can be described in a natural fashion by $\Sigma$. In this paper it is shown that a necessary and sufficient condition for a congruence scheme $\Sigma$ whose polynomials do not contain constants to have a representation is that each polynomial in the sequence be at least binary.

1. Introduction. For an algebra $\mathfrak{A}$ and $a, b \in A$, let $\Theta(a, b)$ denote the smallest congruence relation under which $a \equiv b$; such relations are called principal. For instance, in the class $\boldsymbol{D}$ of distributive lattices (see [1]):

$$
\begin{aligned}
c \equiv d(\Theta(a, b)) \text { iff } & \\
c & =p_{0}(b, a, b, c, d) \\
p_{0}(a, a, b, c, d) & =p_{1}(a, a, b, c, d) \\
p_{1}(b, a, b, c, d) & =p_{2}(b, a, b, c, d) \\
p_{2}(a, a, b, c, d) & =p_{3}(a, a, b, c, d) \\
p_{3}(b, a, b, c, d) & =d,
\end{aligned}
$$

where

$$
\begin{aligned}
& p_{0}\left(x, y_{0}, y_{1}, y_{2}, y_{3}\right)=\left(\left(x \wedge y_{0}\right) \vee y_{2}\right) \wedge\left(y_{2} \vee y_{3}\right), \\
& p_{1}\left(x, y_{0}, y_{1}, y_{2}, y_{3}\right)=\left(x \vee y_{0} \vee y_{2}\right) \wedge\left(y_{2} \vee y_{3}\right), \\
& p_{2}\left(x, y_{0}, y_{1}, y_{2}, y_{3}\right)=\left(\left(x \vee y_{0}\right) \wedge y_{2}\right) \vee y_{3}, \\
& p_{3}\left(x, y_{0}, y_{1}, y_{2}, y_{3}\right)=\left(x \wedge y_{0} \wedge y_{2}\right) \vee y_{3} .
\end{aligned}
$$

This is one example of a congruence scheme (for a general definition, see $\S 2$ ). The general definition of a congruence scheme permits an arbitrary sequence $p_{0}, \cdots, p_{n-1}$ of polynomials and the polynomials may have any number of variables.

As the simplest example of a congruence scheme, let $\boldsymbol{K}$ be an equational class and let us assume that for all $\mathfrak{A} \in \boldsymbol{K}$ the following holds:

$c \equiv d(\Theta(a, b))$ iff $c=a+e$ and $d=b+e$ for some $e \in A$, where + is a binary operation of $\boldsymbol{K}$.

Congruence schemes have been investigated in [5] under the name 1-good systems and in [1]. 
An equational class $\boldsymbol{K}$ is a uniform representation of a congruence scheme $\Sigma$ iff the principal congruence relations in all $\mathfrak{A} \in \boldsymbol{K}$ can be described by $\Sigma$. The paper [1] starts with an equational class $K$ representing a congruence scheme $\Sigma$ and considers the consequences of this assumption.

In this paper we start with a congruence scheme $\Sigma$ and ask under what conditions there is a nontrivial equational class $K$ representing $\Sigma$. A trivial necessary condition (see $\S 2$ ) is that each polynomial be at least binary. However, this is not sufficient, as exemplified by the scheme $0+((0+x)+y)$ (see $\S 5$ ). Our main result is that the above condition is necessary and sufficient, provided the polynomials in the scheme contain no nullary operations.

In $\S 2$ we give all the basic definitions. In $\S 3$ the representation theorem is reduced to finding a suitable simple algebra. The construction of this simple algebra is presented in $\S 4$. Some additional results, examples, and problems are given in $\S 5$.

2. Basic definitions. We assume that the reader is familiar with the basic concepts of universal algebra; see [2].

Given a set of polynomials $\Omega$ (of a given type $\tau$ ) and a class of algebras $\boldsymbol{K}$ (of type $\mu$ ) we say that $\boldsymbol{K}$ admits $\Omega$ or that the type of $\Omega$ is contained in the type of $\boldsymbol{K}$ iff all operation symbols occurring in $\Omega$ are operations in $\boldsymbol{K}$, in other words, if every $p \in \Omega$ can be regarded a polynomial of $\boldsymbol{K}$.

Now we give the three basic definitions. The reader should be familiar with Mal'cev's lemma (see, e.g., Theorem 10.3 in [2]), which forms the background for these definitions.

Definition 1. For a type $\tau$ of algebras, a congruence scheme (briefly, scheme) $\Sigma$ of type $\tau$ is a sequence $p_{0}, \cdots, p_{n-1}$ of polynomials of type $\tau$, where $p_{i}$ is a polynomial in the variables $x$ and $y_{j}^{i}, 0 \leqq$ $j<n_{i}$, together with a function $t:\{0,1, \cdots, n-1\} \rightarrow\{0,1\}$.

We shall say that a class $\boldsymbol{K}$ of algebras admits $\Sigma$ if $\boldsymbol{K}$ admits the polynomials in $\Sigma$.

REMARKs. The variables $x$ and $y_{0}^{i}\left(0 \leqq j<n_{i}\right)$ are assumed to occur explicitly in $p_{i}$. Variables with distinct indices (upper or lower) are distinct from each other and from $x$. The integer $n_{i}$ can be 0 , in which case the only variable of $p_{i}$ is $x$. (Some easy changes are necessary in the example given in the introduction to make it satisfy the formal requirements of Definition 1.)

Definition 2. Let $\Sigma$ be a congruence scheme and $\mathfrak{A}$ an algebra admitting $\Sigma$. We define a 4 -ary relation on $A$ : 
For $a_{0}, a_{1}, b_{0}, b_{1} \in A,\left\langle a_{0}, a_{1}, b_{0}, b_{1}\right\rangle$ is in the relation $\Sigma$, in symbols, $\Sigma\left(a_{0}, a_{1}, b_{0}, b_{1}\right)$, iff there are elements $c(i, j) \in A\left(0 \leqq i<n, 0 \leqq j<n_{i}\right)$ satisfying the following equations:

(i ) $b_{0}=p_{0}\left(a_{t(0)}, c(0,1), \cdots, c\left(0, n_{0}-1\right)\right)$,

(ii) $\quad p_{i}\left(a_{1-t(i)}, c(i, 0), \cdots, c\left(i, n_{i}-1\right)\right)=p_{i+1}\left(a_{t(i+1)}, c(i+1,0), \cdots\right.$, $\left.c\left(i+1, n_{i+1}-1\right)\right), i=0,1, \cdots, n-2$,

(iii) $\quad p_{n-1}\left(a_{1-t(n-1)}, c(n-1,0), \cdots, c\left(n-1, n_{n-1}-1\right)\right)=b_{1}$.

REMARK. We call the $c(i, j)$ the elements used to establish $\Sigma\left(a_{0}, a_{1}, b_{0}, b_{1}\right)$. Of course, in general there are many such $c(i, j)$.

Now we can restate Mal'cev's lemma in terms of these definitions:

Lemma 3. Let $\mathfrak{A}$ be an algebra. For $a, b, c, d \in A, c \equiv d(\Theta(a, b))$ iff there exists a congruence scheme $\Sigma$ (of the same type as that of I) such that $\Sigma(a, b, c, d)$.

DEFinition 4. Let $K$ be a class of algebras (of the same type) and let $\Sigma$ be a congruence scheme. $K$ is a representation of $\Sigma$, in notation, $\boldsymbol{K} \vDash \Sigma$, iff $\boldsymbol{K}$ admits $\Sigma$ and for all $\mathfrak{A} \in \boldsymbol{K}$ and $a, b, c, d \in A$,

$$
c \equiv d(\Theta(a, b)) \quad \text { iff } \quad \Sigma(a, b, c, d) \text { holds in } \mathfrak{A} .
$$

If $\boldsymbol{K}=\{\mathfrak{A}\}$, then we say that $\mathfrak{A}$ is a representation of $\Sigma$ or $\mathfrak{A} \vDash \Sigma$.

REmaRK. For $a, b \in A$, then $b \equiv b(\Theta(a, a))$ always holds. Thus if $\mathfrak{A} \vDash \Sigma$, then we always have $\Sigma(a, a, b, b)$; similarly, we can also conclude $\Sigma(a, b, a, b)$ and $\Sigma(a, b, b, a)$.

Definition 5. A congruence scheme $\Sigma$ has a uniform representation $K$ iff $K$ is a nontrivial equational class representing $\Sigma$.

For examples, the reader is referred to [1].

The following observation will be useful:

COROLLARY 6. Let $\mathfrak{A}$ be an algebra admitting the congruence scheme $\Sigma$. For $a, b \in A$, let $c(i, j)$ be the elements used to establish $\Sigma(a, a, b, b)$. Then

$$
p_{i}\left(a, c(i, 0), \cdots, c\left(i, n_{i}-1\right)\right)=b, \quad \text { for all } 0 \leqq i<n .
$$

Proof. The polynomials exhibited in Definition 2 with the substitution of $a, a, b, b$ are all congruent to each other and to $b$ under $\Theta(a, a)=\omega$, hence the statement.

Now we come to the necessary condition. 
LEMMA 7. Let $\Sigma$ be a congruence scheme. If $n_{i}=0$ for some $0 \leqq i<n$, then $\Sigma$ has no representation by an algebra with more than one element. In particular, $\Sigma$ has no uniform representation.

Proof. Let $\mathfrak{A} \vDash \Sigma$ and $|A|>1$. Let $n_{i}=0$. Let $a, b \in A$ and $a \neq b$. By Definition $4, \Sigma(\alpha, a, b, b)$ and so by Corollary $6, p_{i}(\alpha)=b$. Similarly, $\Sigma(a, a, a, \alpha)$ and hence $p_{i}(a)=a$, contradicting that $a \neq b$.

3. The reduction theorem. The proof of the main theorem is given in two stages.

THEOREM 8. Let $\Sigma$ be a congruence scheme. Then $\Sigma$ has a uniform representation iff there exists a simple algebra with more than one element representing $\Sigma$.

Proof. Let us assume that $\Sigma$ has a uniform representation, that is, $\boldsymbol{K} \vDash \Sigma$, where $\boldsymbol{K}$ is a nontrivial equational class. By a result of R. Magari [4], $\boldsymbol{K}$ contains a simple algebra $\mathfrak{A}$ with more than one element. Since $\mathfrak{A} \in \boldsymbol{K}, \mathfrak{A} \vDash \Sigma$.

Conversely, let $\mathfrak{A}$ be a simple algebra of type $\mu$ with more than one element and let $\mathfrak{A} \vDash \Sigma$. Let $\mu^{\prime}$ denote the type we obtain by augmenting $\mu$ with a ternary operation $q$ and the 4 -ary operations $q(i, j), 0 \leqq i<n, 0 \leqq j<n_{i}$.

Since $\mathfrak{A}$ is simple and $\mathfrak{A} \vDash \Sigma, \Sigma\left(a_{0}, a_{1}, b_{0}, b_{1}\right)$ holds in $\mathfrak{A}$ whenever $a_{0} \neq a_{1}$ or if $a_{0}=a_{1}$ and $b_{0}=b_{1}$. Let $c(i, j)$ denote the elements used to establish $\Sigma\left(a_{0}, a_{1}, b_{0}, b_{1}\right)$.

Define

$$
q(i, j)\left(a_{0}, a_{1}, b_{0}, b_{1}\right)=c(i, j) .
$$

Define $q(i, j)\left(\alpha_{0}, a_{0}, b_{0}, b_{1}\right)$ for $b_{0} \neq b_{1}$ in an arbitrary way.

Let $q$ be an arbitrary majority function on $A$, that is, a ternary function satisfying $q(a, b, b)=q(b, b, a)=q(b, a, b)=b$ for all $a, b \in A$.

Let $\mathfrak{H}^{\prime}$ denote the algebra of type $\mu^{\prime}$ we obtain from $\mathfrak{A}$ by defining $q$ and the $q(i, j)$ as described above.

Let $p_{i}^{\prime}$ denote the polynomial defined by

$$
p_{i}^{\prime}=p_{i}\left(x, q(i, 0)\left(z_{0}^{i}, z_{1}^{i}, z_{2}^{i}, z_{3}^{i}\right), \cdots, q\left(i, n_{i}-1\right)\left(z_{0}^{i}, z_{1}^{i}, z_{2}^{i}, z_{3}^{i}\right)\right) .
$$

Then $\mathfrak{A}^{\prime}$ satisfies the following set $\Phi$ of first-order axioms:

$$
\begin{aligned}
& (x)(y)(q(x, y, y)=q(y, x, y)=q(y, y, x)=y) \\
& \left(x_{0}\right)\left(x_{1}\right)\left(y_{0}\right)\left(y_{1}\right)\left(x_{0} \neq x_{1} \longrightarrow\left(y_{0}=p_{0}^{\prime}\left(x_{t(0)}, x_{0}, x_{1}, y_{0}, y_{1}\right) \wedge \cdots \wedge\right.\right. \\
& p_{i}^{\prime}\left(x_{1-t(i)}, x_{0}, x_{1}, y_{0}, y_{1}\right)=p_{i+1}^{\prime}\left(x_{t(i+1)}, x_{0}, x_{1}, y_{0}, y_{1}\right) \wedge \cdots \wedge
\end{aligned}
$$




$$
\begin{aligned}
& \left.\left.p_{n-1}\left(x_{1-t(n-1)}, x_{0}, x_{1}, y_{0}, y_{1}\right)=y_{1}\right)\right) \\
& (x)(y)\left(y=p_{0}^{\prime}(x, x, x, y, y)=\cdots=p_{n-1}^{\prime}(x, x, x, y, y)\right)
\end{aligned}
$$

Let $\boldsymbol{K}_{1}$ denote the class of all algebras of type $\mu^{\prime}$ satisfying $\Phi$. Since $\mathfrak{U}^{\prime} \in \boldsymbol{K}_{1}, \mathbb{K}_{1}$ contains an algebra of more than one element. $\boldsymbol{K}_{1}$ is closed under the formation of ultraproducts (since $\boldsymbol{K}_{1}$ is axiomatic), subalgebras (since $\Phi$ is universal), and homomorphic images (since all algebras in $K_{1}$ are simple, in view of the second sentence in $\Phi$ and the fact that $\boldsymbol{K}_{1}$ contains the one-element algebra). In view of the first sentence in $\Phi$, all algebras in $\boldsymbol{K}=\boldsymbol{H} \boldsymbol{S P}\left(\boldsymbol{K}_{1}\right)$ are congruence distributive, hence by Jónsson's lemma (see, e.g., [2]) and the observations in the previous sentence, $\mathbb{K}$ is the class of subdirect products of algebras in $\boldsymbol{K}_{1}$.

Finally, we verify $\mathfrak{B} \models \Sigma$ for all $\mathfrak{B} \in \mathbb{K}$. Since $\mathfrak{B} \in \mathbb{K}, \mathfrak{B}$ is a subdirect product of algebras $\mathfrak{A}_{m} \in \boldsymbol{K}_{1}, m \in \mathbb{M}$. Take $a_{0}, a_{1}, b_{0}, b_{1} \in B$ satisfying $b_{0} \equiv b_{1}\left(\Theta\left(a_{0}, a_{1}\right)\right)$. For $x \in B$, let $x^{m}$ denote the projection of $x$ into $\mathfrak{A}_{m}$. Then $b_{0}^{m} \equiv b_{1}^{m}\left(\Theta\left(a_{0}^{m}, a_{1}^{m}\right)\right)$ for all $m \in M$. Thus

$$
\Sigma\left(a_{0}^{m}, a_{1}^{m}, b_{0}^{m}, b_{1}^{m}\right)
$$

is established with the elements $q(i, j)\left(a_{0}^{m}, a_{1}^{m}, b_{0}^{m}, b_{1}^{m}\right)$. Let $c(i, j)$ denote the element of $\Pi\left(\mathfrak{A}_{m} \mid m \in M\right)$ for which

$$
c(i, j)^{m}=q(i, j)\left(a_{0}^{m}, \alpha_{1}^{m}, b_{0}^{m}, b_{1}^{m}\right)
$$

for all $m \in M$. Since $c(i, j)$ is a polynomial of $a_{0}, a_{1}, b_{0}, b_{1}$, we have $c(i, j) \in B$ and so these elements (the $c(i, j), 0 \leqq i<n, 0 \leqq j<n_{i}$ ) establish $\Sigma\left(a_{0}, a_{1}, b_{0}, b_{1}\right)$ in $\mathfrak{B}$. This completes the proof of Theorem 8 .

Observe that the equational class $\boldsymbol{K}$ constructed to prove Theorem 8 has a number of interesting properties: $\boldsymbol{K}$ is congruence distributive; $\boldsymbol{K}$ is semisimple, that is, all subdirectly irreducible algebras in $\boldsymbol{K}$ are simple; $\mathbb{K}$ has the Congruence Extension Property (this follows from a result in [1]). By adding more operations in the construction of $\mathfrak{U}^{\prime}$ we could make $\boldsymbol{K}$ satisfy any Mal'cev condition, such as congruence permutability, regularity, and so on.

4. The construction of simple algebras. To construct a simple algebra representing a given congruence scheme $\Sigma$, we first prove two theorems about idempotent algebras and a special type of con. gruence scheme. An algebra $\mathfrak{A}$ is called idempotent iff all operations $f$ of $\mathfrak{U}$ have arity $>0$ and $f(a, \cdots, a)=a$ for all $a \in A$. A polynomial $p$ is called idempotent reduced iff it contains no constants and no subpolynomials of the form $f(u, \cdots, u)$ where $f$ is an operation. For instance, $\left(x+y_{1}\right)+\left(\left(x+y_{1}\right)+y_{2}\right)$ is idempotent reduced, while

$$
\left(x+\left(y_{1}+y_{2}\right)\right)+\left(x+\left(y_{1}+y_{2}\right)\right) \text { and }\left(x+\left(y_{1}+y_{1}\right)\right)+\left(x+y_{2}\right)
$$


are not.)

THEOREM 9. Let $\mathfrak{A}$ be an idempotent algebra of type $\tau,\langle a, b, c, d\rangle \in$ $A^{4}$ and either $a \neq b$ or $a=b$ and $c=d$. Let $p\left(x, y_{1}, \cdots, y_{n}\right)$ be an idempotent reduced polynomial of type $\tau, n \geqq 1$. Then there exists an idempotent algebra $\mathfrak{X}^{\prime}$ containing $\mathfrak{A}$ as a subalgebra and there are elements $e_{1}, \cdots, e_{n} \in A^{\prime}$ such that $c=p\left(a, e_{1}, \cdots, e_{n}\right)$ and $d=$ $p\left(b, e_{1}, \cdots, e_{n}\right)$.

Proof. With a polynomial $p$ we associate a partial algebra $\mathbb{C}(p)$. First we define $C(p)$ :

$$
\begin{aligned}
& \text { for } p=x_{i}, C(p)=\{p\} ; \\
& \text { if } p=f\left(p_{0}, \cdots, p_{n-1}\right), \quad \text { where } f \text { is an operation and }
\end{aligned}
$$

$C\left(p_{0}\right), \cdots, C\left(p_{n-1}\right)$ have already been defined, then

$$
C(p)=\{p\} \cup \cup\left(C\left(p_{i}\right) \mid 0 \leqq i<n\right) .
$$

For every operation $f$ in the type we define a partial operation $f$ on $C(p)$; the domain of $f$ in $C(p)$ consists of all $\left\langle p_{0}, \cdots, p_{n-1}\right\rangle \in$ $C(p)^{n}\left(f\right.$ is $n$-ary) for which $q=f\left(p_{0}, \cdots, p_{n-1}\right) \in C(p)$ and we define $f\left(p_{0}, \cdots, p_{n-1}\right)$ to be $q . \mathbb{S}(p)$ is the corresponding partial algebra.

Now let $\mathfrak{A}, p\left(x, y_{1}, \cdots, y_{n}\right)$, and $a, b, c, d$ be given. First we consider the case $a \neq b$.

Define $D=A \cup\left(C\left(p\left(x, z_{1}, \cdots, z_{n}\right)\right) \cup C\left(p\left(x^{\prime}, z_{1}, \cdots, z_{n}\right)\right)\right)$, where $\cup$ stands for disjoint union and $x, x^{\prime}, z_{1}, \cdots, z_{n}$ are distinct variables. Observe that $C\left(p\left(x, z_{1}, \cdots, z_{n}\right)\right) \cap C\left(p\left(x^{\prime}, z_{1}, \cdots, z_{n}\right)\right)$ consists of all subpolynomials of $p\left(x, z_{1}, \cdots, z_{n}\right)$ that do not contain $x$ while

$$
C\left(p\left(x, z_{1}, \cdots, z_{n}\right)\right) \cap[x]=\{x\} \text { and } C\left(p\left(x^{\prime}, z_{1}, \cdots, z_{n}\right)\right) \cap[x]=\varnothing .
$$

Define on $D$ the operations $f$ of $\mathscr{U}$ as partial operations. If $f$ is $m$-ary, the domain of $f$ in $D$, Dom $(f, \mathfrak{I})$, is

$$
A^{m} \cup \operatorname{Dom}\left(f, \mathfrak{E}\left(p\left(x, z_{1}, \cdots, z_{n}\right)\right)\right) \cup \operatorname{Dom}\left(f, \mathfrak{E}\left(p\left(x^{\prime}, z_{1}, \cdots, z_{n}\right)\right)\right)
$$

and $f$ is defined on its domain to make $\mathfrak{A}, \mathfrak{S}\left(p\left(x, z_{1}, \cdots, z_{n}\right)\right)$ and $\mathfrak{C}\left(p\left(x^{\prime}, z_{1}, \cdots, z_{n}\right)\right)$ subalgebras of $\mathfrak{D}$. It is easily seen that this definition is not ambiguous.

Now let $\Theta$ be the smallest equivalence relation on $D$ under which $a \equiv x, b \equiv x^{\prime}, c \equiv p\left(x, z_{1}, \cdots, z_{n}\right)$, and $d \equiv p\left(x^{\prime}, z_{1}, \cdots, z_{n}\right)$. We claim that $\Theta$ is a congruence relation of $\mathfrak{D}$. Indeed, let $f$ be an $m$-ary partial operation, let $u_{i} \equiv v_{i}(\Theta), i=1,2, \cdots, m$, and let $f\left(u_{1}, \cdots, u_{m}\right)$, $f\left(v_{1}, \cdots, v_{m}\right)$ be both defined. Since $\Theta$ restricted to $A$ is $\omega$, we can assume without loss of generality that 


$$
\left\langle u_{1}, \cdots, u_{n}\right\rangle \in \operatorname{Dom}\left(f, \mathfrak{S}\left(p\left(x, z_{1}, \cdots, z_{n}\right)\right)\right) .
$$

Since $p$ is not a proper subpolynomial of itself, no $u_{i}$ is $p\left(x, z_{1}, \cdots, z_{n}\right)$. Hence either $\left\langle v_{1}, \cdots, v_{n}\right\rangle \in A^{n}$ and

$$
\left\langle u_{1}, \cdots, u_{n}\right\rangle=\langle x, \cdots, x\rangle,\left\langle v_{1}, \cdots, v_{n}\right\rangle=\langle a, \cdots, a\rangle,
$$

contradicting that $p$ is idempotent reduced or

$$
\left\langle v_{1}, \cdots, v_{n}\right\rangle \in \operatorname{Dom}\left(f, \mathfrak{S}\left(p\left(x, z_{1}, \cdots, z_{n}\right)\right)\right)
$$

in which case we must have $u_{i}=v_{i}$ for all $i=1, \cdots, m$ since $\Theta$ restricted to $C\left(p\left(x, z_{1}, \cdots, z_{n}\right)\right)$ is $\omega$. This proves that $\Theta$ is a congruence relation.

Now let $\mathfrak{X}^{\prime}$ be any completion of $\mathfrak{D} / \Theta$ into an idempotent algebra.

If $a=b$, then we start with $D=A \cup C\left(p\left(x, z_{1}, \cdots, z_{n}\right)\right)$ and proceed as above. This completes the proof of Theorem 9 .

Let us call a congruence scheme $\Sigma$ idempotent reduced iff each polynomial occurring in $\Sigma$ is idempotent reduced.

THEOREM 10. Let $\Sigma$ be an idempotent reduced congruence scheme satisfying $n_{i} \geqq 1$ for $0 \leqq i<n$. Then there exists a countable nontrivial simple idempotent algebra $\mathfrak{A}$ representing $\Sigma$.

Proof. We proceed by repeated applications of Theorem 9. Let $\mathfrak{A}_{0}$ be an idempotent algebra of a type admitting $\Sigma$ and $1<\left|A_{0}\right| \leqq \boldsymbol{\aleph}_{0}$ (such an $\mathfrak{A}_{0}$ obviously exists). Now let us assume that we already have $\mathfrak{A}_{0}, \cdots, \mathfrak{A}_{m}$ with the following properties:

(i) $\mathfrak{A}_{i-1}$ is a subalgebra of $\mathfrak{A}_{i}$ for $i<m$; (ii) if $\left\langle a_{0}, a_{1}, b_{0}, b_{1}\right\rangle \in A_{i-1}$ and $a_{0} \neq a_{1}$ or $a_{0}=a_{1}$ and $b_{0}=b_{1}$, then $\Sigma\left(a_{0}, a_{1}, b_{0}, b_{1}\right)$ in $\mathfrak{A}_{i}$ for $i \leqq m$; (iii) $\left|A_{i}\right| \leqq \aleph_{0}$ for $i \leqq m$. To construct $\mathfrak{A}_{m+1}$ let

$$
\left\{\left\langle a_{0}^{i}, a_{1}^{i}, b_{0}^{i}, b_{1}^{i}\right\rangle \mid 0<i<\omega\right\}
$$

be all elements $\left\langle a_{0}, a_{1}, b_{0}, b_{1}\right\rangle$ of $A_{m}^{4}$ such that $a_{0} \neq a_{1}$ or $a_{0}=a_{1}$ and $b_{0}=b_{1}$ and $\Sigma\left(a_{0}, a_{1}, b_{0}, b_{1}\right)$ does not hold in $\mathfrak{A}_{m}$. We define a sequence of idempotent algebras $\mathfrak{B}_{0}, \mathfrak{B}_{1}, \cdots, \mathfrak{B}_{k} \cdots k<\omega$ such that $\mathfrak{B}_{0}=\mathfrak{A}_{m}, \mathfrak{B}_{i}$ is a subalgebra of $\mathfrak{B}_{i+1},\left|B_{i}\right| \leqq \aleph_{0}$, and $\Sigma\left(\alpha_{0}^{i}, a_{1}^{i}, b_{0}^{i}, b_{1}^{i}\right)$ in $\mathfrak{B}_{i}$. Indeed, if $\mathfrak{B}_{0}, \cdots, \mathfrak{B}_{i}$ are already constructed, then we construct $\mathfrak{B}_{i+1}$ as follows: if $\Sigma\left(a_{0}^{i+1}, a_{1}^{i+1}, b_{0}^{i+1}, b_{1}^{i+1}\right)$ in $\mathfrak{B}_{i}$, then $\mathfrak{B}_{i}=\mathfrak{B}_{i+1}$. If not, we adjoin to $B_{i} n-1$ new elements $w_{0}, \cdots, w_{n-2}$ and take an idempotent algebra $\mathfrak{B}_{i}^{\prime}$ containing $\mathfrak{B}_{i}$ as a subalgebra, $\left|\boldsymbol{B}_{i}^{\prime}\right| \leqq \boldsymbol{K}_{0}$, such that $w_{0}, \cdots, w_{n-2} \in$ $B_{i}^{\prime}$. Now successively apply (the construction of) Theorem 9 to $\left\langle a_{t(0)}^{i}, a_{1-t(0)}^{i}, b_{0}^{i}, w_{0}\right\rangle,\left\langle a_{t(1)}^{i}, a_{1-t(1)}^{i}, w_{0}, w_{1}\right\rangle, \cdots,\left\langle a_{t(n-1)}^{i}, a_{1-t(n-1)}^{i}, w_{n-2}, b_{1}^{i}\right\rangle$ and call the resulting algebra $\mathfrak{B}_{i+1}$. Then $\left|B_{i+1}-B_{i}\right|<\aleph_{0}$, hence $\left|B_{i+1}\right| \leqq$ *o.

Let $\mathfrak{A}_{m}$ be the direct limit of the $\mathfrak{B}_{i}, i<\omega$, and let $\mathfrak{A}$ be the 
direct limit of $\mathfrak{A}_{m}, m<\omega . \quad \mathfrak{A}$ is obviously idempotent and nontrivial, and $|A| \leqq \aleph_{0}$. To show that $\mathfrak{A}$ is simple we verify that for $a, b, c, d \in$ $A$ and $a \neq b$, we have $c \equiv d(\Theta(a, b))$. We prove more: if $\left\langle a_{0}, a_{1}, b_{0}, b_{1}\right\rangle \in$ $A^{4}$ and $a_{0} \neq a_{1}$ or $a_{0}=a_{1}$ and $b_{0}=b_{1}$, then $\Sigma\left(a_{0}, a_{1}, b_{0}, b_{1}\right)$. This is obvious, since $\left\langle a_{0}, a_{1}, b_{0}, b_{1}\right\rangle \in \mathfrak{A}_{m}$ for some $m<\omega$ and hence $\Sigma\left(a_{0}, a_{1}, b_{0}, b_{1}\right)$ in $\mathfrak{A}_{m+1}$ and therefore in $\mathfrak{A}$. This shows that $\mathfrak{A}$ is simple and that $\mathfrak{A}$ represents $\Sigma$. This completes the proof of Theorem 10 .

To obtain the main result we have to get rid of the condition "idempotent reduced" in the previous results.

Definition 11. Define a map $p \rightarrow \hat{p}$ from the set of polynomials containing no constants of a given type into itself by the rules:

(i) $\hat{x}=x$ for any variable $x$.

(ii) Let $p=f\left(q_{1}, \cdots, q_{m}\right)$, where $f$ is an operation. Then $\hat{p}=$ $\hat{q}_{1}$, if $\widehat{q}_{1}=\cdots=\widehat{q}_{m}$ and $\hat{p}=f\left(\hat{q}_{1}, \cdots, \hat{q}_{m}\right)$ otherwise.

For a congruence scheme $\Sigma, \hat{\Sigma}$ is the scheme obtained from $\Sigma$ by replacing each polynomial $p$ by $\hat{p}$ (and retaining $t$ ).

CoRollary 12. (i) Let $p$ be a polynomial. If the variable $x$ occurs in $p$, then it occurs in $\hat{p}$.

(ii) Let $\mathfrak{A}$ be an idempotent algebra admitting the polynomial p. Then $p\left(a_{1}, \cdots, a_{n}\right)=\hat{p}\left(a_{1}, \cdots, a_{n}\right)$ for all $a_{1}, \cdots, a_{n} \in A$.

(iii) Let $\mathfrak{A}$ be an idempotent algebra and let $\Sigma$ be a congruence scheme. Then $\mathfrak{A}$ represents $\Sigma$ iff $\mathfrak{A}$ represents $\hat{\Sigma}$.

Now our main result is a simple combination of Lemma 7, Theorems 8, 10, and Corollary 12(iii):

THEOREM 13. A congruence scheme $\Sigma$ containing no constant has a uniform representation iff all polynomials in $\Sigma$ are at least binary.

This construction can be easily modified to give a single uniform representation of any set of congruence schemes satisfying the hypothesis of Theorem 13.

5. Concluding remarks. We start out with a scheme containing a constant which does not have a uniform representation even though it satisfies the necessary condition of Lemma 7 . In fact, we prove more.

THEOREM 14. Let $\Sigma$ be the congruence scheme given by $p=$ $0+((0+x)+y)$ and $t(0)=0$. Then there is no algebra $\mathfrak{A}$ with more than one element representing $\Sigma$. 
Proof. Let $\mathfrak{A}$ represent $\Sigma,|A|>1$. We claim that $x \rightarrow 0+x$ is a permutation of $A$. Indeed, let $a, b \in A, 0+a=0+b$, and $c \equiv$ $d(\Theta(a, b))$ in $\mathcal{A}$. Then $\Sigma(a, b, c, d)$, that is, $c=0+((0+a)+e)$ and $d=0+((0+b)+e)$, hence $c=d$. Thus $\Theta(a, b)=\omega$ and so $a=b$. Since $a \equiv a(\Theta(0,0))$, there is an $e \in A$, such that $a=0+((0+a)+e)$, hence $a=0+a^{\prime}$ with $a^{\prime}=(0+a)+e$. This shows that to every $a \in A$, there is a unique $a^{\prime} \in A$ satisfying $a=0+a^{\prime}$.

Next fix an $a \in A, a \neq 0^{\prime}$ and consider the congruence $a \equiv$ $b\left(\Theta\left(0^{\prime}, a\right)\right)$. We claim that $b$ is uniquely determined. Indeed, $\Sigma\left(0^{\prime}, a, a, b\right)$, hence $a=0+\left(\left(0+0^{\prime}\right)+e\right)$ and $b=0+((0+a)+e)$. In the first equation, $0+0^{\prime}=0$, hence $a=0+(0+e)$. Thus $0+e=a^{\prime}$ and so $e$ is uniquely determined (in fact, $e=a^{\prime \prime}$ ), yielding $b=0+$ $\left((0+a)+a^{\prime \prime}\right)$. Thus $b$ is uniquely determined. But this is clearly a contradiction since both $b=a$ and $b=0^{\prime}$ satisfy the congruence.

Binary polynomials play a special role in connection with representations of congruence schemes as witnessed by the following results:

(i) Let the scheme $\Sigma$ have a uniform representation. Then there exists a binary scheme $\Sigma^{\prime}$ (that is, all polynomials in $\Sigma^{\prime}$ are binary) such that $\Sigma^{\prime}$ has a uniform representation and every equational class $K$ representing $\Sigma^{\prime}$ also represents $\Sigma$.

(ii) An equational class representing a binary congruence scheme contains no finite algebra of more than one element.

(iii) Any equational class $\boldsymbol{K}$ representing a congruence scheme satisfies an identity of the form $x=q(x, y)$, where $q$ is a binary polynomial in which $x$ and $y$ occur. In particular, $\boldsymbol{K}$ cannot be regular.

(ii) is the most complicated of these three statements so we shall prove it. Let $\Sigma$ be a congruence scheme with the polynomials $p_{i}\left(x, y_{i}\right), 0 \leqq i<n$. Let $\mathfrak{A}$ be an algebra, $\mathfrak{A} \vDash \Sigma$, and let $A$ be finite, $|A|>1$. Fix $a_{0}, a_{1} \in A, a_{0} \neq a_{1}$. For all $b \in B$ we have $\Sigma\left(a_{0}, a_{0}, b, b\right)$ and $\Sigma\left(a_{1}, a_{1}, b, b\right)$; let $f_{i}^{\circ}(b), 0 \leqq i<n$, and $f_{i}^{1}(b), 0 \leqq i<n$, be the elements establishing these. By Corollary 6,

$$
p_{i}\left(a_{j}, f_{i}^{j}(b)\right)=b, j=0,1 ; i=0,1, \cdots, n-1 .
$$

Thus each $f_{i}^{j}$ is one-to-one. Since $A$ is finite, each $f_{i}^{j}$ is a permutation of $A$. In particular, each $f_{i}^{j}$ is onto, hence

$$
p_{i}(x, u)=p_{i}(x, v) \text { implies that } u=v .
$$

Consider now any $b \in A$ satisfying $\Sigma\left(a_{0}, a_{1}, a_{0}, b\right)$, and let $c_{0}, \cdots$, $c_{n-1}$ be the elements used to establish it. Thus

$$
\begin{aligned}
a_{0} & =p_{0}\left(a_{t(0)}, c_{0}\right), \cdots, p_{i}\left(a_{1-t(i)}, c_{i}\right) \\
& =p_{i+1}\left(a_{t(i+1)}, c_{i+1}\right), \cdots, p_{n-1}\left(a_{1-t(n-1)}, c_{n-1}\right)=b .
\end{aligned}
$$


Thus $c_{0}$ is uniquely determined, in fact, $c_{0}=f_{0}^{t(0)}\left(a_{0}\right)$. Similarly, $c_{1}=f_{1}^{t(1)}\left(p_{0}\left(a_{1-t(0)} c_{0}\right)\right)$. Continuing thus, we find that each $c_{i}, 1 \leqq i \leqq n$, is uniquely determined and so $b$ is uniquely determined. But this is clearly a contradiction since both $b=a_{0}$ and $b=a_{1}$ satisfy $\Sigma\left(a_{0}, a_{1}\right.$, $\left.a_{0}, b\right)$ and $a_{0} \neq a_{1}$. This completes the proof of (ii).

(ii) is particularly interesting because it provides a large variety of equational classes $\boldsymbol{K}$ of algebras with no nontrivial finite algebras. In fact our method finds such $\boldsymbol{K}$ that are idempotent, congruence distributive, etc.

Another result of some interest is

(iv) Let $\Sigma$ be a congruence scheme with $n=1$ (that is, with a single polynomial). Then every equational class representing $\boldsymbol{K}$ is congruence permutable.

This follows from $\mathrm{H}$. Lakser [3]; see also $\mathrm{H}$. Werner [6].

Some of the open problems can be stated in terms of three quasi-orderings defined on congruence schemes:

$$
\begin{aligned}
\Sigma_{0} \subseteq_{i} \Sigma_{1} & \Longleftrightarrow\left(\Sigma_{0}(a, b, c, d) \Longrightarrow \Sigma_{1}(a, b, c, d)\right) \\
\Sigma_{0} \subseteq_{a} \Sigma_{1} & \Longleftrightarrow\left(\mathfrak{A} \vDash \Sigma_{0} \Longrightarrow \mathfrak{A} \vDash \Sigma_{1}\right) \\
\Sigma_{0} \subseteq_{e} \Sigma_{1} & \Longleftrightarrow\left(\boldsymbol{K} \vDash \Sigma_{0} \Longrightarrow \boldsymbol{K} \models \Sigma_{1}\right), \\
& \text { where } \boldsymbol{K} \text { is an equational class. }
\end{aligned}
$$

Natural problems are:

I. Describe the relations $\subseteq_{i}, \subseteq_{a}, \subseteq_{e}$.

II. Define $\Sigma_{0} \equiv_{i} \Sigma_{1}$ iff $\Sigma_{0} \subseteq_{i} \Sigma_{1}$ and $\Sigma_{1} \subseteq_{i} \Sigma_{0}$, similarly define $\equiv_{a}$ and $\equiv_{e}$. Given a scheme $\Sigma$, find a scheme $\Sigma^{\prime}$ of smallest "rank" such that $\Sigma^{\prime} \equiv_{i} \Sigma$; similarly for $\equiv_{a}$ and $\equiv_{a}$.

III. It would be interesting to investigate equational classes of semigroups and some other algebraic systems form the point of view of congruence schemes.

The main result of this paper is no longer true if in Definition 1 a $p_{i}$ and a $p_{j}(i \neq j)$ can have more than one variable in common.

IV. Find the corresponding result.

Rather than using a scheme to describe principal congruences, one can devise schemes to describe $\mathrm{V}\left(\Theta\left(a_{i}, b_{i}\right) \mid i=1, \cdots, n\right)$.

V. Generalize the main result to such schemes.

Given an algebra $\mathfrak{A}$, define $\operatorname{Sch} \mathfrak{A}$ as the set of all congruence schemes $\Sigma$ such that $\mathfrak{A} \vDash \Sigma$. For a class $\boldsymbol{K}$ of algebras, set 
$\operatorname{Sch} \boldsymbol{K}=\bigcap(\operatorname{Sch} \mathfrak{A} \mid \mathfrak{A} \in \boldsymbol{K})$.

Observe that $\subseteq_{a}$ and $\subseteq_{e}$ can be defined in terms of Sch $\mathfrak{A}$ and Sch $\boldsymbol{K}$, respectively.

This notation justifies the use of the term "representation" since $\boldsymbol{K}$ represents $\Sigma$ iff $\Sigma \in \operatorname{Sch} \boldsymbol{K}$, that is, $\Sigma$ actually occurs in Sch $\boldsymbol{K}$.

\section{REFERENCES}

1. E. Fried, G. Grätzer and R. Quackenbush, Uniform Congruence Schemes.

2. G. Grätzer, Universal Algebra, Van Nostrand, Princeton, N. J., 1968.

3. H. Lakser, Principal congruences in permutable equational classes.

4. R. Magari, Una dimonstrazione del fatto che ogni varietà ammette algebre semplici, Ann. Univ. Ferrara, 14 (1969), 1-4.

5. - The classification of idealizable varieties (Congruenze Ideali IV), J. of Algebra, 26 (1973), 152-165.

6. H. Werner, A Mal'cev condition for admissible relations, Algebra Univ., 3 (1973), 263.

Received December 21, 1976 and in revised form September 10, 1977. The research of both authors was supported by the National Research Council of Canada. The main result of the paper was announced in the abstract, Notices Amer. Math. Soc., 23 (1976), A156.

University of Illinois at Chicago Circle

Chicago, IL 60680

AND

UNIVERSITY OF MANITOBA

WINNIPEG, MANITOBA

CANADA R3T 2N2 



\section{PACIFIC JOURNAL OF MATHEMATICS}

\section{EDITORS}

RICHARD ARENS (Managing Editor)

University of California

Los Angeles, CA 90024

Charles W. Curtis

University of Oregon

Eugene, OR 97403

C. C. MOORE

University of California

Berkeley, CA 94720

\section{J. DugundJI}

Department of Mathematics

University of Southern California

Los Angeles, CA 90007

R. FinN and J. Milgram

Stanford University

Stanford, CA 94305

\section{ASSOCIATE EDITORS}
E. F. BECKENBACH
B. H. NeumanN
F. WOLF
K. YosHIDA

\section{SUPPORTING INSTITUTIONS}

\author{
UNIVERSITY OF BRITISH COLUMBIA \\ CALIFORNIA INSTITUTE OF TECHNOLOGY \\ UNIVERSITY OF CALIFORNIA \\ MONTANA STATE UNIVERSITY \\ UNIVERSITY OF NEVADA, RENO \\ NEW MEXICO STATE UNIVERSITY \\ OREGON STATE UNIVERSITY \\ UNIVERSITY OF OREGON
}

\author{
UNIVERSITY OF SOUTHERN CALIFORNIA \\ STANFORD UNIVERSITY \\ UNIVERSITY OF HAWAII \\ UNIVERSITY OF TOKYO \\ UNIVERSITY OF UTAH \\ WASHINGTON STATE UNIVERSITY \\ UNIVERSITY OF WASHINGTON
}

The Supporting Institutions listed above contribute to the cost of publication of this Journal, but they are not owners or publishers and have no responsibility for its content or policies.

Mathematical papers intended for publication in the Pacific Journal of Mathematics should be in typed form or offset-reproduced, (not dittoed), double spaced with large margins. Please do not use built up fractions in the text of the manuscript. However, you may use them in the displayed equations. Underline Greek letters in red, German in green, and script in blue. The first paragraph or two must be capable of being used separately as a synopsis of the entire paper. Items of the bibliography should not be cited there unless absolutely necessary, in which case they must be identified by author and journal, rather than by item number. Manuscripts, in triplicate, may be sent to any one of the editors. Please classify according to the scheme of Math. Reviews, Index to Vol. 39. All other communications should be addressed to the managing editor, or Elaine Barth, University of California, Los Angeles, California, 90024.

50 reprints to each author are provided free for each article, only if page charges have been substantially paid. Additional copies may be obtained at cost in multiples of 50 .

The Pacific Journal of Mathematics is issued monthly as of January 1966. Regular subscription rate: $\$ 72.00$ a year (6 Vols., 12 issues). Special rate: $\$ 36.00$ a year to individual members of supporting institutions.

Subscriptions, orders for numbers issued in the last three calendar years, and changes of address should be sent to Pacific Journal of Mathematics, 103 Highland Boulevard, Berkeley, California, 94708. Older back numbers obtainable from Kraus Periodicals Co., Route 100, Millwood, NY 10546.

PUBLISHED BY PACIFIC JOURNAL OF MATHEMATICS, A NON-PROFIT CORPORATION

Printed at Kokusai Bunken Insatsusha (International Academic Printing Co., Ltd.). 8-8, 3-chome, Takadanobaba, Shinjuku-ku, Tokyo 160, Japan.

Copyright (C) 1978 by Pacific Journal of Mathematics Manufactured and first issued in Japan 


\section{Pacific Journal of Mathematics}

\section{Vol. 76, No. $2 \quad$ December, 1978}

Stephanie Brewster Brewer Taylor Alexander, Local and global convexity in complete Riemannian manifolds ...........................

Claudi Alsina i Català, On countable products and algebraic convexifications of probabilistic metric spaces ...............................

Joel David Berman and George Grätzer, Uniform representations of

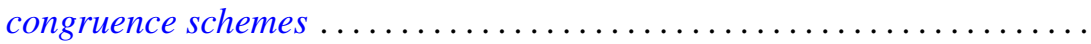

Ajit Kaur Chilana and Kenneth Allen Ross, Spectral synthesis in

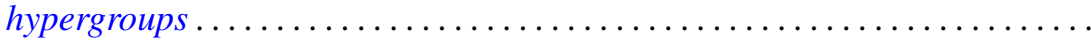

David Mordecai Cohen and Howard Leonard Resnikoff, Hermitian quadratic forms and Hermitian modular forms . .........................

Frank Rimi DeMeyer, Metabelian groups with an irreducible projective

representation of large degree .............................

Robert Ellis, The Furstenberg structure theorem .....................

Heinz W. Engl, Random fixed point theorems for multivalued mappings .......

William Andrew Ettling, On arc length sharpenings ..................

Kent Ralph Fuller and Joel K. Haack, Rings with quivers that are trees........

Kenneth R. Goodearl, Centers of regular self-injective rings ...............

John Gregory, Numerical algorithms for oscillation vectors of second order

differential equations including the Euler-Lagrange equation for

symmetric tridiagonal matrices.

Branko Grünbaum and Geoffrey Shephard, Isotoxal tilings

Myron Stanley Henry and Kenneth Leroy Wiggins, Applications of

approximation theory to differential equations with deviating

arguments

Mark Jungerman, The non-orientable genus of the n-cube .

Robert Richard Kallman, Only trivial Borel measures on $S_{\infty}$ are

quasi-invariant under automorphisms ................

Joyce Longman and Michael Rich, Scalar dependent algebras in the

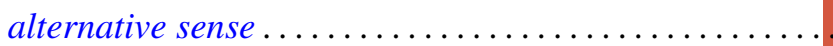

Richard A. Mollin, The Schur group of a field of characteristic zero ........ 471

David Pokrass, Some radical properties of rings with $(a, b, c)=(c, a, b) \ldots 479$

Margaret Shay and Paul Ruel Young, Characterizing the orders changed by

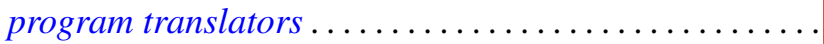

Jerrold Norman Siegel, On the structure of $B_{\infty}(F), F$ a stable space...

Surjeet Singh, (hnp)-rings over which every module admits a basic

submodule...

A. K. Snyder, Universal interpolating sets and the Nevanlinna-Pick property in

Banach spaces of functions...

Jeffrey D. Vaaler, On the metric theory of Diophantine approximation ... 